\title{
Electricity and Magnetism: Insights into the brain from multimodal imaging
}

\author{
M.S. Cohen \\ UCLA Center for Cognitive Neuroscience \\ 760 Westwood Plaza, Suite 17-369 \\ Los Angeles, CA 90095 USA \\ mscohen@ucla.edu
}

\begin{abstract}
The windows into brain function given us by the instruments of neuroimaging each are murky and their view is limited. Simultaneous collection of data from multiple modalities offers the potential to overcome the weaknesses of any tool alone. We argue that the combination of electroencephalography (EEG) and functional magnetic resonance imaging (fMRI) offers observations - and hypothesis testing - not possible using either single instrument.

Because of their safety profiles and their non-invasive natures, EEG fMRI are among the best available devices for the study of human brain. These methods are complementary. EEG is fast, operating in a time domain comparable to single unit activity, but its localizing power is poor and the field of view is limited. While fMRI has the highest spatial resolution of any noninvasive imaging method and can reveal multiple centers of brain activity implicated in cognitive tasks, it is very slow compared to mental activity and is a poor choice for studying rapidly evolving processes.

Here, we address theoretical models of the coupling between EEG and fMRI signals based on cellular physiology and energetics and argue that both tools observe principally synaptic activity. We discuss the technical problems of mutual interference then present several models of brain rhythms for which the joint EEG and fMRI observations provide significant evidence.
\end{abstract}

\section{INTRODUCTION}

“...most neuroscientists reject EEG and MEG evidence in the beliefs that recording wave activity is equivalent to observing an engine with a stethoscope or a computer with a D'Arsonval galvanometer. However, one can learn a lot about a system by listening and watching, if one knows what to seek and find."

-Walter Freeman [1]

"...the classical concept of cerebral localization is of limited value, because of its static character and its failure to provide any answer to the question of how specialized parts of the cortex interact to produce the integration evident in thought or behavior. The problem here is one of the dynamic relations of the diverse parts of the cortex, whether they be cells or cortical fields."

- Karl Lashley [2]

It is well accepted that moment-to-moment signaling in the brain is electrical and that much of the neural code is in the form of action potential spike trains. However, with the exception of a very few examples we are unable to infer from recordings how these phenomena organize to become part of the complex architecture of human sensation or cognition. In humans, it is impractical to record brain action potentials (APs) directly except in extreme settings, such as the development of brain computer interfaces for persons with neuropathic impairments, or in the context of surgery planning in epilepsy and deep brain stimulation. The emergent effects of APs are almost certainly ensemble properties, but only a very few neurons can be sampled at once. Most cognitive processes engage functionally specialized regions throughout the brain, yet direct recordings are highly local in space. Obviously, these direct methods are too invasive to use on healthy volunteers.

\section{ElECTROENCEPHALOGRAPHY (EEG)}

EEG is an attractive alternative for recording electrical activity as it is entirely non-invasive. The phenomenon, discovered over 130 years ago by Caton [3], is that relatively high amplitude $(\sim 100 \mu \mathrm{V})$ signals can be recorded by measuring voltage differentially across the scalp. Cell signaling through synapses creates a current flow between synaptic regions in the dendrites and the cell body or soma as a consequence of membrane conductance changes. In the human cortex the majority of neurons are pyramidal cells [4] with a highly asymmetric morphology that places the cell bodies in deeper layers of the cortex and the dendrites closer to the surface. As a result of the cell shape, current flow between dendrites and soma creates an electrical dipole, albeit a tiny one. The fact that these cells tend to be arranged in parallel arrays oriented normal to the cortical surface allows the dipole moments of the individual cells to interfere constructively, creating a macroscopically detectable field at the scalp (Fig. 1). Further, the time constants of the synaptic potentials are on the order of $100 \mathrm{~ms}$, so that they tend to summate in time. This is thought to be the origin of the EEG. Unfortunately, even though the forward problem (calculating the scalp potentials from

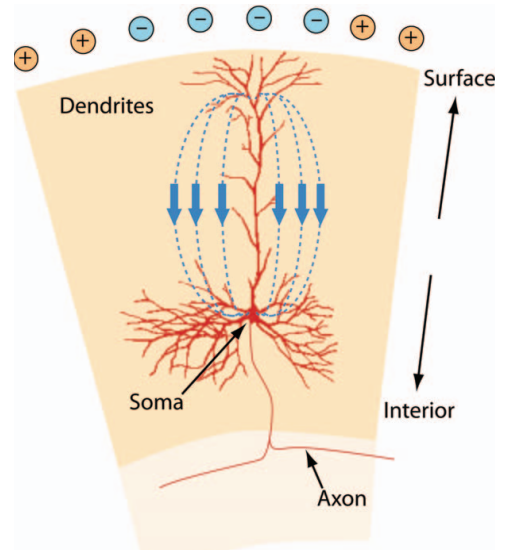


cellular dipoles) is potentially solvable in closed form, the inverse problem is not. Thus it is not possible to uniquely localize the sources that create the scalp potentials from scalp recordings alone.

\section{Functional Magnetic Resonance IMAging (fMRI)}

Functional MRI by the Blood Oxygenation Level Dependent (BOLD) method detects changes in MRI signal that result from variations in venous oxygen concentration. In turn, these are thought to be associated with local variations in energy demand from cell signaling (e.g., [5]). Like EEG, fMRI is a non-invasive method. It has submillimeter spatial resolution and is capable of capturing the entire brain. fMRI is thus well-suited to studying interactions among brain regions. However, the signal changes in fMRI evolve very slowly, with dominant time constants of seconds [6]. Our goal is to simultaneously measure fMRI signal changes and electrical signaling through the EEG (or other means). In so doing, we should be able to identify the sources that create features of the EEG thereby enabling a variety of important research and clinical applications.

\section{Neurovascular COUPLing}

The mechanism by which neuronal signaling is coupled to the BOLD response has been studied extensively [5] and will not be reviewed here. For our purposes it is useful to consider the energetic cost of neuronal activity. Neurons are known to form APs as a result of chemically-mediated transient changes in membrane conductance that occur first at the synapse and then spread throughout the cell and its dendritic and axonal processes. Charged ions run passively down their concentration gradients into and out of the cell creating a typical AP of 1 or $3 \mathrm{~ms}$ in duration and about $130 \mathrm{mV}$ in amplitude. The AP itself, therefore, does not demand energy transduction but relies on the difference in ion concentration inside and outside of the cell, which is maintained by the socalled sodium potassium pump. The latter consumes energy in the form of adenosine tri-phosphate to transport ions actively against their concentration gradient forming a Nernst potential. In considering the relationship between EEG and fMRI it is interesting therefore to look at the energetic costs of signaling.

Assuming a cell volume of about $9 \times 10^{-13} 1$ for an average $30 \mu \mathrm{m}$ neuron, about half of which is liquid, and a sodium concentration of about $40 \mathrm{mM}$, we can calculate that the cell contains about $4 \times 10^{-14}$ Moles of $\mathrm{Na}^{+}$. The AP causes a 130 $\mathrm{mV}$ potential change, $V$, across the cell membrane. Based on the nominal membrane thickness of about $5 \mathrm{~nm}$ we can predict a capacitance, $C$, of about $1 \mu \mathrm{F} / \mathrm{cm}^{2}$. The charge, $Q$, that must move is the product $C V$, about 1.3 coulomb $/ \mathrm{cm}^{2} \approx 1.3 \times 10^{-12}$ Moles $/ \mathrm{cm}^{2}$. For the same neuron, the surface area is about $2.8 \times 10^{-5} \mathrm{~cm}^{2}$ implying that about $3.7 \times 10^{-17}$ Moles of $\mathrm{Na}^{+}$pass with each AP, changing the $\mathrm{Na}^{+}$concentration by about $0.1 \%$. The neuron is thus able to fire hundreds of AP's from the cell's stored potential energy a phenomenon first observed by Baker, et al., when they replaced the entire internal contents of a neuron with salt solution and recorded many thousands of normal appearing AP's [7].

It is more likely then, that the fMRI observations do not relate directly to AP's, but instead to the synaptic events themselves. Some hint of this can be seen in electron micrographs that show high concentrations of mitochondria in the pre- and post-synaptic processes distant from the cell body. Presumably, these provide energy locally to support synaptic function. Although the synaptic energy budget has not been carefully worked out, there are many demands including chemosynthesis and transport, vesicle formation, etc... Strong evidence that the BOLD signal is driven by synaptic activity is given by Logothetis, et al., who recorded neuronal potentials and fMRI concurrently in a monkey model and showed that features of the BOLD signal correlated more strongly to synaptic activity than to AP formation. Most fMRI practitioners today attribute the BOLD signal to synaptic activity [8].

For present purposes, this is of interest because the EEG and fMRI signals apparently derive from the same underlying processes. Consequentially, it is reasonable to imagine that the simultaneous recordings capture many of the same events.

\section{Simultaneous EEG AND fMRI}

Collection of EEG and fMRI at the same time is made challenging because the recording apparatuses themselves interfere destructively, causing large artifacts and signal losses and introducing safety concerns. The principal contamination of the fMRI signal arises from three sources: 1) RF Noise production by the digital electronics (mitigated by shielding), 2) Magnetic field distortion (mitigated by the use of non ferromagnetic electrodes, in our case salt sponges and 3) RF (MRI) signal losses into the EEG leads themselves, mitigated by proper ground return paths and lead dress. A safety issue results from the possibility that large currents introduced into the lead wires can couple to the patient through the low resistance connection to the head, a question addressed below.

The interference that the MRI creates in the EEG is both more complex and more challenging. To form an MR image using the popular echo planar method [9] it is necessary to create large $(2-3$ gauss $/ \mathrm{cm})$ and rapidly varying $(20-50 \mathrm{~T} / \mathrm{s})$ field gradients. By Lenz's law these introduce current in the loops formed by the EEG leads that create voltages thousands of times larger than the EEG itself. An additional problem results from the so-called ballistocardiogram (BCG) - the motion of the body and head that occurs with each impulsive heart contraction; this moves the EEG wires within the strong imaging fields, and it too creates error signals in the EEG recordings. These are of much lower amplitudes (a few $\mu \mathrm{V}$ ) but are irregular in their time course.

Our general solution to the EEG problem is relatively simple, based on the periodic properties of the artifact generators (the MRI scanner and BCG.) We simply assume that over an appropriate interval, $t r$, for the scanner, the artifact is repeated identically and is uncorrelated to the EEG signal onto which it is superposed. Averaging of the contaminated signal thus results in a good estimate of the 
artifact, which can then be subtracted from the contaminated signal leaving only EEG. Letting $S(n)$ represented the set of contaminated samples collected during the $n^{\text {th }} t r$, letting $E E G(n)$ indicate the true EEG signal during that $t r$, and $A$ represent the artifact - which is presumed to be stable recorded over this same interval,

$$
S(n)=E E G(n)+A \text {. }
$$

In turn, $E_{A}(n)$, the estimate of the artifact during the $n^{\text {th }} t r$ is:

$$
E_{A}(n)=\sum_{k=1}^{n} S(k) .
$$

Thus, we should be able to find $\operatorname{EEG}(n)=S(n)-A$. There are a number of problems and challenges with this approach, however.

First, the EEG and scanner artifacts are, in fact, highly correlated because the (loud) sound of the scanner elicits a neural response that appears on the scalp as an evoked potential. The artifact template therefore will contain a representation of the evoked potential as well as the scanner artifact, and this will be removed systematically from the derived signal. Usually, however, this is a desirable effect because the acoustic response to the scanner noise is rarely a topic of research.

Second, the method works only if the summated EEG and artifact are recorded without distortion. Because of the exceedingly poor SNR of the raw signal (about $0.1 \%$ ) this demands extremely high dynamic range in the amplification and recording chain. Most of the energy in the MRI artifact is in the audio band including the frequencies below $100 \mathrm{~Hz}$ that are of considerable interest in the EEG. Thus, low pass filtering is only marginally effective, though it is important for anti-aliasing. Minimizing the size of any effective loops in the EEG leads is helpful [10]. Cancellation by detection of the artifact without connection to the scalp may also be effective (e.g., Kappametrics, Chantilly, VA).

Finally, for the simple average and subtract scheme to work, the artifact must repeat identically each time, otherwise the estimate of the artifact produced by averaging will be inaccurate. While the scanner reproduces these waveforms with extremely high precision, the digitizer in the EEG device may not capture the events at the same relative time each time that they are produced because its clock typically is not synchronous. Even tiny errors of $0.1 \%$ may leave a residual as large as the EEG. It is tempting to invoke the Nyquist theorem and suggest that we can capture the artifact accurately by sampling at somewhat over twice the maximum frequency contained in it. This, however, is false. While the Nyquist theorem applies to signals acquired over infinite time it is often not appreciated that this is not the case when the signal is sampled over a truncated time window.

One way to visualize the problem is to imagine sampling at a high rate of 5 kilosamples/s. From one $t r$ to the next this will allow a maximum time difference, $\Delta t$, relative to the gradient system, of one half of the dwell time: $100 \mu \mathrm{s}$. The gradientinduced artifacts in the EEG are equal to the product of the timing error and the slew rates, which are on the order of 1000 $\mathrm{V} / \mathrm{s}$, so that there might be as much as $0.1 \mathrm{~V}$ difference in measured amplitude from one $t r$ to the next, which is clearly unacceptable as this, in effect, becomes the magnitude of the residual artifact after subtraction. We can reduce the residual artifact by sampling more rapidly, thereby reducing the $\Delta t \times$ dwell product across $t r$ 's. This can be formulated analytically based on the spectral content of the gradient waveform. Assume that its maximum frequency is $\omega$ radians/s. We can think of the $\Delta t$ between successive $t r$ 's as creating a phase difference, $\varphi$, in the sampling of the gradient waveform. The upper bound for $\varphi$ is simply $\omega \Delta t$. If $\varepsilon$ is represents the apparent amplitude of the difference between the artifacts sampled at different times, we can see that:

$$
\begin{aligned}
\varepsilon & =\cos (2 \pi f t)-\cos (2 \pi f t-\varphi) \\
& =\cos (2 \pi f t) \cos (\varphi-1)-\sin (2 \pi f t) \sin \varphi
\end{aligned}
$$

which, for small errors, is approximately linear in $\Delta t$; in the example above, reducing the artifact to the $\mu \mathrm{V}$ range would require sampling in the $10 \mathrm{MHz}$ range which is unrealistically expensive for an EEG device.

The requirement for rapid sampling can be eliminated if we address the phase shift directly, by synchronizing the digital sampling to the MR scanner. It is relatively easy to reduce the error to a few ns in this manner, and this is the basis for our scanner artifact elimination method [11] that reduces the residual artifact to below the resolution of the analog to digital convertor, in effect eliminating it completely.

Small changes in head position during the scan can result in changes in the amplitude and time course of the gradientrelated artifact. Therefore it is desirable in practice to make the gradient filter adaptive. We have done so by creating a leaky averager that applies an exponentially decreasing weight to artifact samples that are more remote in time. The adaptive averager is implemented very simply as:

$$
E_{A}(n)=w E_{A}(n-1)+(w-1) S(n),
$$

where $0<w<1$ is a weighting factor. This filter has minimal computer memory requirements and is easily adjustable. We note that a moving averager is a relatively poor substitute, as the presumably uncorrelated EEG is suppressed in $E_{A}(n)$ only by the square root of the number of iterations included in the average. This residual EEG represents an error term that would be incorporated into the "corrected" EEG signal.

\section{Clinical CONSIDERATIONS}

There is considerable interest in the management of epilepsy using combined EEG and fMRI. Because surgical resection is frequently the best available intervention for this common disorder, it is essential to have a means of identifying the epileptogenic tissue so that the minimum quantity of brain may be removed. In the periods between ictal (seizure) events, the epileptic brain frequently produces spike-like features in the EEG called Interictal Epileptiform Discharges, or IEDs. These IEDs are notoriously difficult to localize based on EEG alone, but several researchers have demonstrated that local BOLD signal changes often occur following each IED [15]. EEG is used also in sleep clinics, brain cancers and coma. 


\section{EEG RHYTHMS AND BOLD}

Among the most prominent features of the EEG are large amplitude oscillations at relatively stable frequencies. Common phenomena are $8-13 \mathrm{~Hz}$ " $\alpha$ " waves, 5-7 Hz " $\theta$ " waves, and others. These presumably are the result of synchronous synaptic events. We can consider the EEG to represent the linear sum of the synchronous $(S A)$ and asynchronous $(A A)$ synaptic activity. The expected EEG magnitude therefore is roughly proportional to $S A+\sqrt{A A}$. The fMRI signal however, presumably is a function of the sum: $|\mathrm{fMRI}|=f(S A+A A)$. What should be our expectation of the relation between these phenomena and BOLD fMRI?
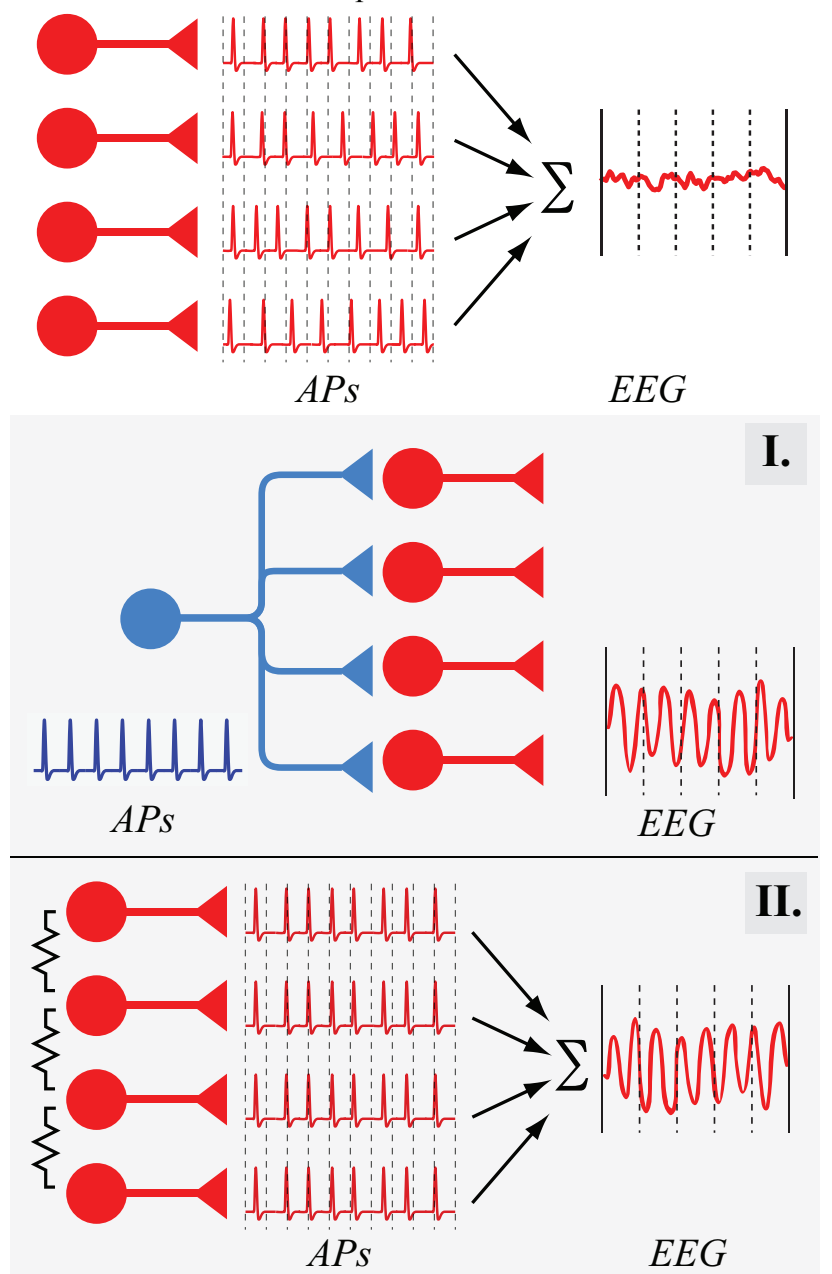

Fig. 2 above presents two plausible models. The top panel suggests that neurons firing asynchronously, at comparable spontaneous rates, produce small amplitude disorganized EEG. Model I (middle) shows a pre-synaptic neuron (blue) driving a set of post-synaptic cells $(r e d)$ to produce a coherent EEG. Model II (bottom) suggests that electrical coupling between the spontaneously firing neurons results in their acting as loosely coupled oscillators that self-synchronize. While model I is energetically costly, predicting a positive BOLD signal, model II is not. Further, model I anticipates that the EEG would become disorganized with ascending synaptic drive, implying that synchronous EEG would be associated with negative BOLD signal. The latter is the reported association between $\alpha$ rhythms and BOLD [12]; we propose that alpha rhythms may arise from the synchronization of pyramidal cell firing that occurs spontaneously in the alpha frequency range. For example, Fig. 3 shows compares the signal intensity in the fMRI scans to the
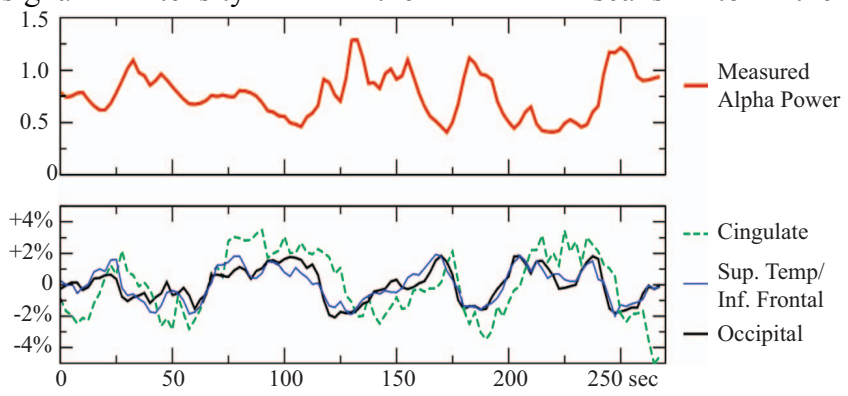

measured alpha band power in a single subject. Sup. Temp. refers to superior temporal cortex and Inf. Frontal indicates inferior frontal cortex. This theory and finding are notably at variance with some classical models [13] that suggest that alpha fluctuations in particular are driven by thalamic pacing. Our data do show a strong positive correlation between thalamic BOLD signal and alpha power. The other scalp rhythms may have either positive or negative BOLD correlations [14].

\section{SOURCE LOCALIZATION}

Although the EEG inverse problem is underspecified, there are a variety of approximation methods for this purpose. We performed concurrent EEG and fMRI experiments using median nerve stimulation to produce cortical evoked responses. Fig. 4 compares dipole localization using BESA (MEGIS Software GmbH, Germany) to conventional fMRI maps made from the same events. Here, BESA identified a

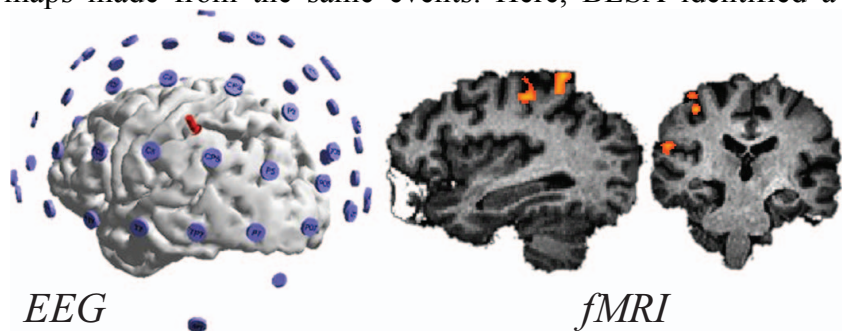

single source (red arrow) on the posterior aspect of the central sulcus. The fMRI data, however, show distinct areas of increased signal near the central sulcus and in insular cortex. There are many possible reasons for this discrepancy; we suggest that the insular engagement, likely representing negative affective valence, is less synchronous and therefore less visible in the scalp EEG. Higher density arrays likely will improve the EEG-based localization.

\section{FUTURE DIRECTIONS}

Our lab is particularly interested in developing the means to record APs and EEG in the human brain during fMRI, as there is little yet known about the specific coupling between directly recorded activity and fMRI signal, except in animal models during sensory stimulation [8]. Further, such a method 
would allow the detection of regions functionally associated with specific units and offer a new window into the systems organization of the brain. Fortunately, the artifact suppression methods outlined above should be equally or even more robust for depth electrodes, where the desired brain signal is much larger. This method is also sampling rate independent, so it is straightforward to increase the bandwidth. To avoid MRI artifacts the electrodes themselves must be made of materials, such as suitably plated copper, with magnetic susceptibility comparable to brain. However, the potential safety problems are extremely serious, as high current densities at the conductive tips of depth electrodes carry the potential to destroy brain tissue. To address this challenge, we studied the antenna properties of the depth electrodes at EEG frequencies and have shown that on resonance the electrodes may cause toxic temperature increases. Fortunately, it is possible to adjust the reactance of the probe so as to bring the device into a safe operating range [16]. As this project developed, it became clear that there advantages to having an antenna deep in the brain, and we have now built and tested a $1 \mathrm{~mm}$ microcoil suitable for such use, collecting both images with resolution better then $100 \mu \mathrm{m}$, as well as spectroscopic data from volumes of only a few $\mathrm{mm}^{3}$ [17]. In the future, we intend to add direct extracellular recording capability to this device.

\section{COnClusions}

Concurrent multimodal recordings of brain activity offer a means to span levels of organization in time and space and to help form a better systems level understanding of the brain. EEG and fMRI are a particularly attractive pair of tools for this work as they are non-invasive and have a long, though separate, history of use. The engineering challenge of simultaneous recording has largely been resolved, so that routine data collection is possible.

The data and theory we presented above suggests that the signal from both methods likely arises from a common process: synaptic transmission, supporting the idea that colocalization is meaningful. The transformation between EEG amplitude and BOLD signal is complicated, however, because the EEG depends on the synchronous activity of many neurons, whereas the BOLD signal does not. Direct experiments using EEG and fMRI to localize brain responses to sensory inputs show both consistency and discrepancy, highlighting the challenge of interpreting such data. Certain prominent EEG features, such as rhythmic waves, may not demand increased energy and therefore might be silent in fMRI studies, or even be anti-correlated. We present a model of spontaneous, cortically generated, alpha rhythms that is consistent with the observation of strong negative correlation between alpha power and fMRI signal.

Combined electrophysiological and MRI recordings are still in their technical infancy, and there is considerable potential to expand to other experiments, such as intracortical recording and microimaging, hopefully opening new and clearer windows into human brain function.

\section{ACKNOWLEDGMENT}

The author wishes to acknowledge Steven Sands and Massoud Akhtari, who collected the data shown in figure 4.

\section{REFERENCES}

[1] W. J. Freeman, "A proposed name for aperiodic brain activity: stochastic chaos," Neural Netw, vol. 13, pp. 11-3, Jan 2000.

[2] K. S. Lashley, "Mass action in cerebral function," vol. 73, pp. 245-254, 1931.

[3] R. Caton, "The electric current of the brain," The British Medical Journal, vol. 2, p. 278, 1875.

[4] N. L. Mitra, "Quantitative analysis of cell types in mammalian neocortex," J Anat, vol. 89, pp. 467-83, Oct 1955.

[5] R. B. Buxton and L. R. Frank, "A model for the coupling between cerebral blood flow and oxygen metabolism during neural stimulation," J Cereb Blood Flow Metab, vol. 17, pp. 64-72, 1997.

[6] M. S. Cohen, "Parametric analysis of fMRI data using linear systems methods," Neuroimage, vol. 6, pp. 93-103, Aug 1997.

[7] P. F. Baker, A. L. Hodgkin, and T. I. Shaw, "Replacement of the axoplasm of giant nerve fibres with artificial solutions," $J$ Physiol, vol. 164, pp. 330-54, Nov 1962.

[8] N. K. Logothetis, J. Pauls, M. Augath, T. Trinath, and A. Oeltermann, "Neurophysiological investigation of the basis of the fMRI signal," Nature, vol. 412, pp. 150-7., 2001.

[9] P. Mansfield, "Multi-planar image formation using NMR spin echoes," $J$ Phys C, vol. 10, pp. L55-L58, 1977.

[10] R. Goldman, J. Stern, J. Engel, and M. S. Cohen, "Acquiring Simultaneous EEG and Functional MRI," Clinical Neurophysiology, vol. 111, pp. 1974-80, Nov 2000.

[11] M. S. Cohen, "Method and apparatus for reducing contamination of an electrical signal," USA: Regents of the University of California, 2003.

[12] R. I. Goldman, J. M. Stern, J. Engel, Jr., and M. S. Cohen, "Simultaneous EEG and fMRI of the alpha rhythm," Neuroreport, vol. 13, pp. 2487-92, Dec 202002.

[13] F. H. Lopes da Silva, J. E. Vos, J. Mooibroek, and A. Van Rotterdam, "Relative contributions of intracortical and thalamo-cortical processes in the generation of alpha rhythms, revealed by partial coherence analysis," Electroencephalogr Clin Neurophysiol, vol. 50, pp. 449-56, 1980.

[14] E. Martinez-Montes, P. A. Valdes-Sosa, F. Miwakeichi, R. I. Goldman, and M. S. Cohen, "Concurrent EEG/fMRI analysis by multiway Partial Least Squares," Neuroimage, vol. 22, pp. 1023-34, Jul 2004.

[15] K. Krakow, Cross, G., Symms, M., woermann, F., Allen, P., Fish, D., Duncan, J., "Reproducable Localization of intericital activation in patients with folacl epilepsy by using spike-trigger fMRI," in Epilepsia, 1998, p. 199.

[16] D. Strick, M. S. Cohen, F. G. Shellock, and J. W. Judy, "Intracranial MR and implant safety," in Society for Neuroscience 37th annual meeting, San Diego, 2007, p. 668.6.

[17] D. S. Strick, R. L. Nunnally, J. C. Smith, W. Clark, D. J. Mills, M. S. Cohen, and J. W. Judy, "Towards a microcoil for intracranial and intraductal MR microscopy," Conf Proc IEEE Eng Med Biol Soc, vol. 2008, pp. 2047-50, 2008. 